Tan, P.E., Yu, P.K., Balaratnasingam, C., Cringle, S.J., Morgan, W.H., McAllister, I.L. \& Yu, D. (2012). Quantitative confocal imaging of the retinal microvasculature in the human retina. Investigative Ophthalmology \& Visual Science, 53(9), 5728-5736

Copyright (c) Association for Research in Vision and Ophthalmology

This is the authors' accepted manuscript version of an article accepted for publication in Investigative Ophthalmology \& Visual Science, following peer review. The version published by the Association for Research in Vision and Ophthalmology is available from the article's abstract page.

This version was made available in the UWA Research Repository on 26 July 2013 in compliance with the publisher's policies on archiving in institutional repositories.

Use of the article is subject to copyright law in respect of the copyright owner. 


\section{QUANTITATIVE CONFOCAL IMAGING OF THE RETINAL MICROVASCULATURE IN THE HUMAN RETINA}

\section{Short title: Microvasculature of the Human Retina}

Word count: 4634

6 Figures

3 Tables

Section code: RE

Authors: Priscilla Ern Zhi Tan ${ }^{1,2}$

Paula K Yu, ${ }^{1,2}$

Chandrakumar Balaratnasingam ${ }^{1,2}$

Stephen J Cringle ${ }^{1,2}$

William H Morgan ${ }^{1}$

Ian L McAllister ${ }^{1}$

Dao-Yi Yu ${ }^{1,2}$

${ }^{1}$ Centre for Ophthalmology and Visual Science, The University of Western Australia, Perth, Australia

${ }^{2}$ The ARC Centre of Excellence in Vision Science, The University of Western Australia, Perth, Australia

Corresponding author:

Professor Dao-Yi Yu

Centre for Ophthalmology and Visual Science and the ARC Centre of

Excellence in Vision Science

The University of Western Australia

Nedlands, Western Australia 6009

Telephone (618) 93810716

Facsimile (618) 93810700

Email_dyyu@lei.org.au

Grant support was provided by the National Health and Medical Research Council of Australia and the Australian Research Council Centre of Excellence in Vision Science

Commercial relationships: $\mathrm{N}$ (all authors) 


\section{$\underline{\text { Abstract }}$}

Purpose: To quantitatively investigate the distribution of blood vessels in different neural layers of the human retina.

Methods: 16 human donor eyes were perfusion-fixed and labelled for endothelial f-actin. Retinal eccentricity located $3 \mathrm{~mm}$ superior to the optic disk was studied using confocal scanning laser microscopy. Immunohistochemical methods applied to whole-mount and transverse sections were used to co-localise capillary networks with neuronal elements. Capillary morphometry, diameter and density measurements were compared between networks.

Results: Four different capillary networks were identified and quantified in the following regions: (1) Nerve fibre layer (NFL). (2) Retinal ganglion cell (RGC) layer. (3) Border of inner plexiform layer (IPL) and superficial boundary of the inner nuclear layer (INL). (4) Boundary of deep INL and outer plexiform layer. The inner most and outermost capillary networks demonstrated a laminar configuration while IPL and deep INL networks displayed a complex three-dimensional configuration. Capillary diameter in RGC and IPL networks were significantly less than other networks. Capillary density was greatest in the RGC network (26.74\%) and was significantly greater than the NFL (13.69\%), IPL (11.28\%) and deep INL (16.12\%) networks.

Conclusions: The unique metabolic demands of neuronal sub-compartments may influence the morphometric features of regional capillary networks. Differences in capillary diameter and density between networks may have important correlations with neuronal function in the human retina. These findings may be important for understanding pathogenic mechanisms in retinal vascular disease. 


\section{$\underline{\text { Introduction }}$}

The precise organisation of vascular structures within the human retina allows the optical properties of the eye, and the nutritional demands of retinal structures, to be simultaneously maintained. A change in capillary network density, in response to altered retinal homeostasis, is likely to modify the refractive properties of the light path to the outer retina. Similarly, changes in capillary morphometry, as a means of improving retinal optics, may compromise nutritional supply to important neuronal elements.

Concepts regarding the role of neurons in modulating regional blood flow, and thereby the local supply of glucose and oxygen, has undergone significant paradigm shifts over the past decade. ${ }^{1}$ Previous researchers favoured the idea that local blood flow was controlled by negative feedback mechanisms however there is now increasing evidence to suggest that neurons and glial cells play a vital role in controlling the capillary circulation. ${ }^{1,2}$

Understanding the morphometric organisation of capillary systems in the human retina, and the relationship they bear to distinct neuronal layers, may increase our understanding of neurovascular coupling mechanisms that are important in retinal homeostasis. ${ }^{2}$ Detailed knowledge of neuron-glial-vascular interactions in the retina may also provide insights into pathogenic mechanisms relevant to retinal vascular diseases.

Oxygen is an essential substrate for retinal energy metabolism and plays a key role in moderating neurovascular coupling mechanisms. ${ }^{1}$ Intra-retinal oxygen distribution and uptake has been precisely quantified in the mammalian retina and the disparities in metabolic activity between somal, dendritic and synaptic retinal compartments have been previously demonstrated. ${ }^{3-8}$ Although it has been known for decades that the multi-layered retinal capillary network underlies the distribution of oxygen tension in the retina ${ }^{9}$ the relationships between capillary network morphometry and the heterogeneous metabolic demands of neuronal elements remains unclarified. Gariano et al. ${ }^{10}$ have documented the location of 
vascular plexuses within the primate retina and have performed detailed quantitative measurements of the temporal and spatial sequence of vascular plexus formation. These studies have been critical for understanding physiological mechanisms that govern retinal angiogenesis and vascular development. Delineating the morphometric characteristics of capillary networks may identify important vascular adaptations that allow the unique metabolic demands of each retinal layer to be satisfied. It may also allow important correlations between capillary network morphometry and previously determined retinal oxygen measurements to be performed, thus allowing speculation concerning structurefunction relationships in the primate retina. Finally, it may elucidate structural mechanisms that facilitate the fine balance between optical clarity and cellular nutrition in the human eye. In this report we utilise novel, micro-pipette and antibody-based perfusion methodology, together with confocal microscopic techniques, to examine the retinal circulation. ${ }^{11-13}$ The region of retina $3 \mathrm{~mm}$ superior to the optic disk was examined and the morphometric features of capillary networks, respective to distinct retinal layers, were quantified. A region superior to the optic disk was chosen for examination in an effort to avoid the macula - an area with vascular and cellular specialisations that is markedly different to most other retinal eccentricities. ${ }^{14}$ This study is a detailed quantitative assessment of capillary networks that comprise the human retinal circulation and is an important extension to previous studies that have studied retinal vascular characteristics. ${ }^{10,15-17}$

\section{Materials and Methods}

This study was approved by the human research ethics committee at The University of Western Australia. All human tissue was handled according to the tenets of the Declaration of Helsinki. 


\section{Human Donor Eyes}

A total of 16 eyes from 14 donors were studied. All eyes were obtained from the Lions Eye Bank (Lions Eye Institute, Western Australia). Eyes were received after removal of corneal buttons for transplantation. None of the donors had a known history of eye disease. The demographic data, cause of death and post-mortem time to eye perfusion for each donor are presented in Table 1. 12 eyes were used for quantitative analysis, three eyes were used for co-localisation studies and one used for epoxy sectioning (Table 1).

\section{Perfusion Labelling of Retinal Circulation}

Our previous publications have provided a detailed review of the perfusion-based techniques used for targeted retinal endothelial labelling. ${ }^{12,13}$ Identical methodology was used for the present study. Briefly, the central retinal artery was cannulated and residual blood was washed out with oxygenated Ringer's solution and 1\% bovine serum albumin. After the 20minute Ringer's wash, $4 \%$ paraformaldehyde in $0.1 \mathrm{M}$ phosphate buffer was perfused for at least 30 minutes to achieve fixation. $0.1 \%$ Triton-X-100 in $0.1 \mathrm{M}$ phosphate-buffered solution was then perfused for 5 to 7 minutes to aid in the permeation of endothelial cell membranes. Detergent was subsequently washed out by a 30 minute perfusion with $0.1 \mathrm{M}$ phosphatebuffered solution. Microfilaments and cell nuclei were then labelled over the course of 2 hours by using a mixture of phalloidin conjugated to Alexa Fluor 546 (30U; Invitrogen, Carlsbad, CA) and bisbenzamide (H33258; $1.2 \mu \mathrm{g} / \mathrm{ml}$; Sigma-Aldrich, St. Louis, MO) or iodide dye (YO-PRO-1; 6.6 $\mathrm{MM}$; Invitrogen). Residual label was cleared from the vasculature by further perfusion with $0.1 \mathrm{M}$ phosphate buffer. The posterior chamber was then immersion fixed in 4\% paraformaldehyde overnight. 


\section{Tissue Preparation and Immunolabelling}

The posterior globe was dissected at the equator to allow viewing of the posterior retina. The retina was carefully dissected around optic disc edge. A few cuts were made in the peripheral retina to enable the retina to lie flat.

Retinas from two separate donors (Table 1) underwent whole-mount immunolabelling using the protocol described by Xiao and Hendrickson. ${ }^{18}$ This permitted co-localisation of capillary networks relative to distinct retinal layers. In brief, the whole retina was washed in $0.1 \mathrm{M}$ phosphate buffer on a shaker for one to two days, floated in $1 \%$ sodium borohydride in phosphate buffer overnight, cryoprotected in $30 \%$ sucrose and stored at $-80^{\circ} \mathrm{C}$. The retina was subsequently thawed and maintained at $4^{\circ} \mathrm{C}$ on a slow speed shaker prior to immunolabelling. The retina was permeabilised overnight in solution comprising $0.1 \%$ sodium azide and $0.1 \%$ Triton-X 100 in $0.1 \mathrm{M}$ phosphate buffer. The solution was then changed to a blocking serum comprising $10 \%$ normal goat serum in phosphate buffer for overnight incubation. Primary antibodies were then added to the solution for incubation over three to four days. Excess antibodies were washed off by overnight incubation in $0.1 \mathrm{M}$ phosphate buffer. The retina was then incubated with secondary antibodies and nucleic acid label overnight and washed thoroughly in solution for at least 2 hours prior to mounting. Retinal ganglion cells and their axons were labelled using rabbit anti- $\gamma$-synuclein ${ }^{19}$ (1:200, Abcam ab55424) and goat anti-rabbit secondary antibody conjugated to Alexa Fluor 488 (1:200, Invitrogen A11034). For identification of ON-bipolar cells ${ }^{20}$ and horizontal cells ${ }^{20}$ the primary antibodies that were used included mouse anti-Go- $\alpha$ (1:100, Millipore MAB3073) and rabbit anti-parvalbumin (1:200, Swant PV 25) respectively. Secondary antibodies that were conjugated to these primary antibodies included goat anti-mouse antibody (Alexa Fluor 488;1:200, Invitrogen A10680) and goat anti-rabbit antibody (Alexa Fluor 635;1:200, Invitrogen A21070) respectively. Separate retinas were used for labelling 
retinal ganglion cells and horizontal cells as the secondary antibodies for both primary antibodies were derived from rabbit species.

One eye which did not undergo perfusion-labelling was used for immunolabelling colocalisation after frozen sectioning (Table 1). The same retinal eccentricity was examined. Transverse retinal frozen sections, $12 \mu \mathrm{m}$ in thickness, were dried onto Gold slides (Erie Superfrost PLUS, ERIFT-4981GLPLUS-006E; Biolab, Decatur, GA) and used for immunolabelling. Blood vessels were labelled using lectins from Triticum Vulgaris conjugated to TRITC (1:50; Sigma L5266). Nuclei were counter-labelled using bisbenzamide (H33258; 1.2 $\mu \mathrm{g} / \mathrm{ml}$; Sigma-Aldrich, St. Louis, MO). Remaining primary and secondary antibodies that were used for co-localisation studies in these transverse sections were the same as that described above for whole-mount specimens.

\section{Microscopy}

Whole-mount retinas were imaged using low magnification epifluorescence microscopy (E800; Nikon, Tokyo, Japan) before confocal imaging. Epifluoresecene microscopy images were used for precise location of the retinal region, centred $3 \mathrm{~mm}$ superior to the optic disk (Fig. 1), prior to confocal microscopy studies. Such measures ensured that retinal eccentricity used for capillary morphometric comparisons was consistent in each eye donor. Two Nikon C1 microscopes equipped with either three lasers (wavelengths $405 \mathrm{~nm}, 488 \mathrm{~nm}$ and $532 \mathrm{~nm}$ ) or four lasers (wavelengths $405 \mathrm{~nm}, 488 \mathrm{~nm}, 545 \mathrm{~nm}$ and $637 \mathrm{~nm}$ ) were used for confocal imaging. Both microscopes were equipped with EZ-C1 software (v.3.20). Objective lenses that were used for confocal imaging included x4 (NA 0.2), x10 (NA 1.45) and x20 (NA 0.75) Plan-Apo lenses. Using a motorised stage, a series of z-stacks were captured for each specimen beginning from the vitreal surface, at the level of the inner limiting membrane, to the outer retina. Each z-stack consisted of a depth of optical sections collected at $0.35 \mu \mathrm{m}$ increments along the z-plane. Images of different wavelengths were acquired sequentially. 


\section{Image Preparation}

ImagePro Plus (Media Cybernetics, Version 7.1) and Image J (version 1.43, available free online, National Institute of Health, USA, http:/rsb.info.nih.gov/ij) were the image-analysis software that was used to quantify confocal microscope images. All images for the manuscript were prepared using Adobe Photoshop (version 12.1, Adobe Systems Inc.) and Adobe Illustrator CS5 (version 12.1.0, Adobe Systems Inc.).

\section{Study of Capillary Topography}

Morphometric criteria previously used to define vascular plexuses in central nervous system and ocular studies were employed to divide the retinal circulation into different capillary networks. ${ }^{14-16,21}$ A change in capillary branching pattern ${ }^{22,23}$ and projected orientation ${ }^{14,23,24}$ was used to distinguish the different capillary networks. Additionally, using the moviesequence function available on Image $\mathrm{J}$ software to sequentially view the z-stack, it was possible to determine if capillary networks displayed a laminar, single-planar orientation that was predominantly confined to a single retinal layer or if they displayed a complex threedimensional configuration that traversed the z-axis. In this report, the term laminar refers to capillary configurations that are predominantly confined to a single plane with minimal projections along the z-axis. Likewise, the term three-dimensional refers to capillary networks that are not confined to a single plane but instead demonstrate prominent projections along the vertical z-axis.

The movie-sequence function on Image $\mathrm{J}$ was also used to view capillary morphology simultaneously with nuclei and thereby locate the position of capillary networks within the retina. Transverse and flat-mount immunolabelling, with multiple antibodies, was also used to confirm the position of capillary networks relative to the nerve fibre layer (NFL), retinal ganglion cell (RGC) layer, bipolar cells and horizontal cells. 
Quantitative Study of Capillary Networks

A z-projected image of each capillary network together with previously defined histological

parameters, ${ }^{7,13,16}$ was used to perform quantitative measurements. The following

measurements were attained from each network as illustrated in Figure 2:

1. Capillary diameter - Defined as the perpendicular distance across the maximum chord axis of each vessel. Each image was partitioned into 9 equal areas and measurements were acquired from each area to ensure representative sampling.

2. Capillary density - Defined as percentage of the sample area occupied by vessel lumens.

Statistical Analysis

All data is expressed in terms of mean and standard error and were calculated using Sigmastat (Sigmastat, ver. 3.1; SPSS, Chicago, IL). Multiple measurements from eyes with data taken from right and left eyes of the same individual were analyzed using $R$ ( $R$ Foundation for Statistical Computing, Vienna, Austria). ${ }^{25}$ One-way analysis of variance (ANOVA) testing was performed to compare measurements between layers. The model used included "Right" or "Left" nested within "eye donor" as random effects using linear mixed modeling to test measurement differences between retinal layers. ${ }^{25}$ The assignment of donor as a random effect was used to account for the effects of intra-"eye" correlation and similarly "Right" and "Left" to account for right and left eye correlation. Statistical analysis also determined if age accounted for differences in capillary diameter and vascular density measurements between retinal layers. We tested the influence of age upon density using a linear mixed model incorporating age as the predictive factor and right and left eye as random factors to account for variation between right and left eye nested within donor eyes as another random factor. We also performed a one-way ANOVA testing the effect of cause of death 
upon vascular density using the same random effects model as described above. Cause of death was defined as either accidental or cancer.

\section{$\underline{\text { Results }}$}

General

The mean donor age was $49.71 \pm 5.19$ years. We examined 11 left and 5 right eyes from 12 male and 2 female donors. The mean post mortem time to initial perfusion ranged from 3 to 22 hours with an average of $13.39 \pm 1.68$ hours.

All orders of the retinal microvasculature were well perfused after cannulation of the central retinal artery. Endothelial cells, smooth muscle cells and nuclei of the retinal vasculature were clearly labelled.

\section{Capillary Topography in the Human Retina}

Morphometrically different capillary networks were consistently identified in the following locations: (1) Nerve fibre layer. (2) Retinal ganglion cell layer. (3) Border of inner plexiform layer (IPL) and superficial boundary of the inner nuclear layer (INL). (4) Boundary of deep INL and outer plexiform layer (OPL).

Capillaries in the NFL network were orientated parallel to the direction of RGC axon bundles and arose from branch vessels in the RGC layer. Capillaries in the NFL had linear trajectories with fewer inter-capillary anastomotic connections compared to other networks (Fig. 3). Colocalisation using $\gamma$-synuclein antibody confirmed that the location of this network was within the NFL. Within the retinal eccentricity examined in this study there was an observed reduction in capillary density in the NFL as the distance away from the optic disk increased (Fig. 3A).

In the RGC layer (Fig. 4), retinal arterioles were clearly observed to give rise to capillary networks. Retinal venules were also observed in this layer of the retina. Analysis of capillary trajectory in the RGC layer, using the movie-sequence function on Image J, 
demonstrated a complex vascular configuration. Many capillaries in the RGC layer projected along a single plane while some capillaries were observed to run obliquely at varying angles along the z-plane and form anastomoses with capillary networks in the NFL and outer retina. The configuration of capillaries in the RGC network was significantly more threedimensional than the NFL network. Capillary density was also observed to be greatest in the RGC capillary network.

The capillary network between the IPL and superficial portion of the INL also displayed a three-dimensional vascular configuration (Fig. 5). However, capillary density in this layer appeared significantly less than the RGC layer with a pronounced reduction in the number of vessels that projected along a single plane. Co-localisation studies using ON-bipolar cell marker demonstrated that capillaries in this layer were situated amongst bipolar cell processes.

The deepest capillary network was found at the level of horizontal cells in the INL (Fig. 6). Co-localisation studies using Go- $\alpha$ antibody revealed that synapses of ON-bipolar cells were distributed among the capillary network. Similar to the NFL network, capillaries in this layer predominantly demonstrated a laminar configuration with most capillaries projecting along a single plane. Multiple closed loops were also observed in this capillary network.

\section{Quantitative Analysis of Capillary Diameter}

Mean capillary diameter for all networks was $8.26 \pm 0.03 \mu \mathrm{m}$. Table 2 provides mean capillary diameter for each capillary network. Capillary diameter in NFL network was significantly greater than RGC $(P<0.001)$ and IPL networks $(P<0.001)$. Capillary diameter in deep INL network was also significantly greater than RGC $(P<0.001)$ and IPL $(P<$ $0.001)$ networks. There was no difference in capillary diameter between NFL and deep INL networks $(P=0.227)$ and RGC and IPL networks $(P=0.740)$. Age did not influence capillary diameter in any of the networks (all $P>0.050)$. 
Table 3 provides mean capillary diameter for patients that deceased from cancer or accidental means. There was no difference in capillary diameter between the two groups in RGC network $(P=0.303)$, NFL network $(P=0.874)$, IPL network $(P=0.964)$ and deep INL network $(P=0.183)$.

\section{Quantitative Analysis of Capillary Density}

Table 2 provides mean density measurements for each capillary network. Capillary densities were significantly different between networks. Capillary density was greatest in the RGC network and was significantly greater than the NFL network $(P<0.001)$, IPL network $(P<$ $0.001)$ and deep INL network $(P<0.001)$. Capillary density was lowest in the IPL network and was significantly lower than the deep INL network $(P<0.001)$. NFL capillary density was also greater than IPL network $(P=0.036)$. Age did not influence capillary density in any of the networks (all $P>0.050$ ).

Table 3 provides mean capillary density for patients that deceased from cancer and accidental means. There was no difference in capillary density between the two groups in RGC network $(P=0.660)$, NFL network $(P=0.886)$ and IPL network $(P=0.455)$. Capillary density was significantly greater in the cancer group in the deep INL network $(P=0.046)$.

\section{$\underline{\text { Discussion }}$}

Capillary networks in the human retina are required to support the immense energy demands of neuronal components without compromising the optical integrity of the light pathway to the outer retina. In this regard, the function served by the retinal circulation is significantly different to other capillary networks in the central nervous system (CNS). Oxygen tension and demands within the retina is markedly heterogeneous ${ }^{9}$ and the metabolic demands of distinct retinal layers are most likely satisfied, to varying extents, by capillary adaptations that function to increase the efficiency of regional nutrient delivery and waste removal. Studying morphometric variations between capillary networks may therefore provide vital 
information concerning the energy requirements of regional neuronal structures via effective neurovascular coupling mechanisms. It may also allow useful structure-function extrapolations between capillary morphometry and previously determined measurements on retinal metabolism ${ }^{3-8,26-29}$ to be performed.

Previous authors have employed a variety of different techniques to document the organisation of capillary networks in the primate retina. Trypsin digest ${ }^{30}$ and corrosion casting $^{31}$ techniques have provided valuable information concerning the three-dimensional morphology of capillary networks, however, inadvertent tissue damage as a result of these techniques limited localisation of capillary networks respective to retinal layers. Fluorescein angiography $^{32}$ and magnetic resonance imaging ${ }^{33}$ have recently been employed to study the retinal circulation however the limited resolution offered by these techniques again precluded study at a cellular level and restricted co-localisation. A major advantage of the methodology employed in the present study is that it allows complete labelling of the retinal microcirculation without inadvertently altering surrounding non-vascular structures. Triplelabelling of post-perfused tissue also allowed accurate identification of capillary network location within the retina and confirmed what was demonstrated on flat-mount confocal microscope images.

There have been varying reports concerning the order and number of capillary networks in the human retina. Early trypsin digest studies by Toussaint et al..$^{34}$ demonstrated a lack of capillary lamination whilst Michaelson et $a l^{35}$ demonstrated a two-layered laminar pattern using benzidine peroxidase techniques. The histological details reported in these studies were most likely limited to some extent by the microscopic and immunohistochemical techniques that were available at the time. Snodderly et al. ${ }^{15,16}$ and Gariano et al. ${ }^{10}$ published excellent studies in the 1990s and demonstrated two inner and two outer capillary beds in human and non-human primate retinae. Our findings reaffirm that of the latter 2 groups and 
demonstrate four morphometrically different capillary networks in the human retina being present in the following regions: (1) NFL. (2) RGC layer. (3) Border of IPL and superficial boundary of INL. (4) Boundary of deep INL and OPL. Similar to these previous authors ${ }^{10,15,16}$ we found that the innermost and outermost capillary networks displayed a single, planar configuration while that of the RGC and IPL demonstrated a complex threedimensional configuration. Three-dimensional vascular configurations are believed to increase the efficiency of oxygen transfer and waste removal in metabolically active tissues. $^{21,23}$ The variation in retinal capillary network morphology identified in the present study demonstrates important parallels to the human cerebral cortex where the microcirculation is adapted in accordance with regional neuronal demands. ${ }^{24,36,37}$ Previous researchers have demonstrated that the inner $1 \mathrm{~mm}$ of cerebral cortex demonstrates large meshes, the next $2 \mathrm{~mm}$ is filled with fine polygonal meshes and the outer $0.1 \mathrm{~mm}$ contains large quadrangular meshes that run parallel to the surface. ${ }^{24,36,37}$ Taken together our findings suggest that, similar to the brain, capillary networks in the retina are morphometrically adapted to serve the unique functional demands of each retinal layer.

Utilising confocal microscope techniques and image analysis software we were able to quantify the morphometric characteristics of each capillary network and thus extend on the observations made by previous authors. We identified significant differences in capillary diameter between all four networks with the smallest capillary diameter being present in RGC and IPL networks. Mean capillary diameter is one measure of the rate at which a capillary network is able to exchange oxygen per unit volume of blood. ${ }^{38}$ A reduction in capillary diameter increases surface area/blood volume ratio resulting in greater oxygen exchange area for a given amount of blood. In the brain, differences in intra-cortical capillary network diameters correlate with regional variations in neuronal function. ${ }^{39}$ Lower mean diameters of capillary networks in RGC and IPL layers suggest high rates of oxygen 
exchange in this region of the retina. These morphometric findings correlate with our previous in vivo functional studies where we demonstrated high oxygen uptake in the IPL. ${ }^{9}$ Capillary diameter measurements in the present study were also greater than previous reports ${ }^{15,16}$ and may reflect inter-species and tissue preparation differences.

Within the grey matter of the CNS an increase in capillary density correlates strongly with an increase in blood flow and mitochondrial activity. ${ }^{40}$ Histological studies have shown significant variation in vascular density across neuronal layers in the brain. ${ }^{41}$ Total capillary density in the non-human primate fovea, peri-fovea and peri-papillary region has been measured as $40 \%, 45 \%$ and $60-70 \%$ respectively. ${ }^{15,16}$ Vascular density is also known to vary depending on the eccentricity from central retina. ${ }^{10}$ To our knowledge, there have not been previous capillary density measurements of individual networks in the human retina. In this study, capillary density was greatest in the RGC layer implicating it as a metabolically intense region. The dependency of RGCs on a high density capillary circulation may be one reason why this subset of neurons could be particularly vulnerable to acute, transient and mild hypoxic stress. ${ }^{42}$

Although this study provides important new knowledge regarding retinal capillary topography in human eyes we acknowledge several limitations of the report. Firstly, the sample size of this study is relatively small and consists of only 16 human eyes. It is difficult to acquire human eyes from healthy individuals and we did not wish to waste human tissue by performing an extensive analysis when appropriate statistical tests permitted us to reliably identify differences between capillary networks, despite the relative small sample size. The other limitation of this work is that only one retinal eccentricity was examined. The aim of this study was to quantify the morphometric characteristics of capillary networks and speculate upon structure-function relationships between vascular units and regional metabolic activity. It is expected that the morphometric characteristics of capillary networks will vary 
according to retinal eccentricity especially in specialised regions of the retina such as the fovea, macula and immediate peri-papillary tissue. Similar to the report by Snodderly et $a l{ }^{15,16}$ we observed a change in the NFL capillary network in a proximo-distal direction. Gariano et al. ${ }^{10}$ have demonstrated that deeper vascular layers disappear in the peripheral retina. Examination of the far peripheral retina may therefore possibly demonstrate significant alterations to capillary networks.

This study provides important insights into vascular mechanisms relevant to retinal homeostasis. We demonstrated that mode of death was a significant factor in determining capillary density and it is expected that regional capillary networks will be altered by ocular and systemic disease. It will therefore be important to perform similar studies using diseased human eyes as it may enhance our understanding of capillary-mediated mechanisms in retinal vascular disease.

\section{Acknowledgements}

The authors thank the staff of the Lions Eye Bank of Western Australia, Lions Eye Institute for providing human donor eyes; the staff of DonateWest, the Western Australian agency for organ and tissue donation, who facilitated the recruitment of donors into the study by referral and completion of consent processes; and Dean Darcey for his expert technical assistance. 


\section{$\underline{\text { Legends }}$}

Figure 1 - Region of study. Colour fundus (A) and transverse retinal section (B) from healthy subjects demonstrate the clinical and histological features, respectively, of the retinal eccentricity chosen for capillary morphometry analysis. A region $3 \mathrm{~mm}$ superior to the optic disk (fenestrated box) was examined. NFL = nerve fibre layer, $\mathrm{RGC}=$ retinal ganglion cell, $\mathrm{IPL}=$ inner plexiform layer, $\mathrm{INL}=$ inner nuclear layer, $\mathrm{OPL}=$ outer plexiform layer, $\mathrm{ONL}=$ outer nuclear layer, IS = inner-segments of photoreceptors, OS = outer-segment of photoreceptors, $\mathrm{A}=$ artery and $\mathrm{V}=$ vein. Scale bar $=1000 \mu \mathrm{m}$ (colour fundus) and $50 \mu \mathrm{m}$ (histology).

Figure 2 - Quantification of capillary diameter and density. Confocal images of capillary networks (A) were manually traced (B) prior to quantitative analysis. Each traced image was divided into 9 equal areas (red fenestrated lines) to allow representative sampling of capillary diameter. The perpendicular distance across the maximum chord axis of each vessel was used to measure capillary diameter (green). The area occupied by capillary structures, as a proportion of the total image area, was used as a measure of capillary density and expressed as a percentage. Scale bar $=50 \mu \mathrm{m}$.

Figure 3 - Nerve fibre layer capillary network. Whole-mount confocal microscope image captured from a single laser channel (A) demonstrate the linear trajectory of capillaries and the relative absence of anastamotic connections between vessels. There is a reduction in the density of capillaries as the distance away from optic disk increases (left-to-right direction in image). Merged image with nuclei information (B) demonstrate a paucity of cells in this region. Triple-stained transverse retinal section (C) demonstrates the location of this network within the nerve fibre layer (NFL). Lectins stain endothelium, Hoescht stain nuclei and $\gamma$ synuclein stain retinal ganglion cell $(\mathrm{RGC})$ axons. IPL $=$ inner plexiform layer, $\mathrm{INL}=$ inner nuclear layer, $\mathrm{OPL}=$ outer plexiform layer and $\mathrm{ONL}=$ outer nuclear layer. Scale bar $=50 \mu \mathrm{m}$. 
Figure 4 - Retinal ganglion cell layer capillary network. Whole-mount confocal microscope image captured from a single laser channel (A) demonstrate the high density of capillaries in this network. Arterioles and venules are also observed in addition to capillaries. Merged image with nuclei information (B) demonstrate a high density of cells in this region. Triplestained transverse retinal section (C) demonstrates the location of this network within the retinal ganglion cell (RGC) layer. Lectins stain endothelium, Hoescht stain nuclei and $\gamma$ synuclein stain RGCs. NFL = nerve fibre layer, IPL = inner plexiform layer, $\mathrm{INL}=$ inner nuclear layer, $\mathrm{OPL}=$ outer plexiform layer and $\mathrm{ONL}=$ outer nuclear layer. Scale bar $=50 \mu \mathrm{m}$. Figure 5 - Inner plexiform layer and superficial inner nuclear layer capillary network. Whole-mount confocal microscope image captured from a single laser channel (A) demonstrate the low density of capillaries in this network. Merged image with nuclei information (B) demonstrate a large number of cells in this region. Triple-stained transverse retinal section $(\mathrm{C})$ demonstrates that this network is located at the boundary between the inner plexiform layer (IPL) and inner nuclear layer (INL). Lectins stain endothelium, Hoescht stain nuclei and Go- $\alpha$ stain ON-bipolar cells. NFL = nerve fibre layer, RGC = retinal ganglion cell layer, $\mathrm{OPL}=$ outer plexiform layer and $\mathrm{ONL}=$ outer nuclear layer. Scale bar $=$ $50 \mu \mathrm{m}$.

Figure 6 - Deep inner nuclear layer and outer plexiform layer capillary network. Wholemount confocal microscope image captured from a single laser channel (A) demonstrate the planar configuration of this network with multiple closed capillary loops. Merged image with nuclei information (B) demonstrate a high density of cells in this region. Triple-stained transverse retinal section (C) demonstrates that this network is located at the boundary between the inner nuclear layer (INL) and outer plexiform layer (OPL). Lectins stain endothelium, Hoescht stain nuclei and Parvalbumin stain horizontal cells. NFL $=$ nerve fibre 
layer, $\mathrm{RGC}=$ retinal ganglion cell layer, $\mathrm{IPL}=$ inner plexiform layer and $\mathrm{ONL}=$ outer nuclear layer. Scale bar $=50 \mu \mathrm{m}$. 


\begin{tabular}{lccccc}
\hline Donor ID & Age & Sex & Eye & Cause of death & $\begin{array}{c}\text { Time to } \\
\text { cannulation } \\
\text { (hrs) }\end{array}$ \\
\hline$A$ & 22 & $\mathrm{M}$ & $\mathrm{L}$ & MVA & 15.0 \\
$B$ & 32 & $\mathrm{M}$ & $\mathrm{L}$ & MVA & 20.0 \\
$C$ & 49 & $\mathrm{~F}$ & $\mathrm{~L}+\mathrm{R}$ & Cancer & 5.5 \\
$D$ & 67 & $\mathrm{M}$ & $\mathrm{L}$ & Cancer & 9.5 \\
$E$ & 23 & $\mathrm{M}$ & $\mathrm{L}$ & Suicide & 22.0 \\
$F$ & 66 & $\mathrm{M}$ & $\mathrm{L}+\mathrm{R}$ & Cancer & 15.0 \\
$G$ & 22 & $\mathrm{M}$ & $\mathrm{L}$ & Suicide & 15.0 \\
$H$ & 53 & $\mathrm{M}$ & $\mathrm{L}$ & MVA & 14.0 \\
$I^{*}$ & 60 & $\mathrm{M}$ & $\mathrm{L}$ & Collapse & 18.0 \\
$J^{*}$ & 72 & $\mathrm{M}$ & $\mathrm{R}$ & Drowning & 15.0 \\
$K \dagger$ & 72 & $\mathrm{~F}$ & $\mathrm{~L}$ & Sepsis & 3.0 \\
$L \neq$ & 60 & $\mathrm{M}$ & $\mathrm{R}$ & Liver disease & 3.5 \\
$M$ & 59 & $\mathrm{M}$ & $\mathrm{R}$ & Melanoma & 12.0 \\
$N$ & 39 & $\mathrm{M}$ & $\mathrm{L}$ & Sepsis & 20.0 \\
\hline
\end{tabular}

Table 1 - Human donor details. Age (years), sex $(M=$ male or $F=$ female), side $(\mathrm{L}=$ left or $\mathrm{R}=$ right) cause of death and time to cannulation for each eye donor is provided. Donor eyes that were flat-mounted for co-localisation studies are designated $(*)$, the donor eye that was used for epoxy sectioning is designated $(\dagger)$ and the donor eye that was sectioned transversely for co-localisation studies is designated $(\ddagger) . \mathrm{MVA}=$ motor vehicle accident. 


\begin{tabular}{ccccc}
\hline Capillary network & NFL & RGC & IPL / Superficial INL & Deep INL/OPL \\
\hline Capillary Diameter $(\mu \mathrm{m})$ & $8.47 \pm 0.05(540)$ & $8.01 \pm 0.05(540)$ & $7.99 \pm 0.06(540)$ & $8.56 \pm 0.06(540)$ \\
Capillary Density $(\%)$ & $13.69 \pm 0.01(12)$ & $26.74 \pm 0.01(12)$ & $11.28 \pm 0.01(12)$ & $16.12 \pm 0.01(12)$ \\
\hline
\end{tabular}

Table 2 - Quantitative capillary diameter and density data for each network. Mean and standard error for nerve fibre layer (NFL) network, retinal ganglion cell (RGC) layer network, inner plexiform layer and superficial inner nuclear layer (IPL/superficial INL) network and deep inner nuclear layer and outer plexiform layer (deep INL/OPL) network is provided. Numbers in brackets indicate sample number for each measurement. 


\begin{tabular}{c|cc|ccccc|c}
\hline \multirow{2}{*}{ Capillary Network } & \multicolumn{2}{c}{ NFL } & \multicolumn{2}{c}{ RGC } & \multicolumn{2}{c}{ IPL / Superficial INL } \\
\hline Cause of death & Accidental & Cancer & Accidental & Cancer & Accidental & Cancer & Accidental & Cancer \\
\hline Capillary Diameter $(\mu \mathrm{m})$ & $8.30 \pm 0.08$ & $8.64 \pm 0.08$ & $8.02 \pm 0.07$ & $8.01 \pm 0.07$ & $7.98 \pm 0.08$ & $8.00 \pm 0.08$ & $8.34 \pm 0.08$ & $8.78 \pm 0.08$ \\
Total Capillary Density $(\%)$ & $13.17 \pm 0.01$ & $14.22 \pm 0.01$ & $27.25 \pm 0.02$ & $26.23 \pm 0.02$ & $10.81 \pm 0.01$ & $11.75 \pm 0.01$ & $14.72 \pm 0.01$ & $17.52 \pm 0.00$ \\
\hline
\end{tabular}

Table 3 - Morphometric comparisons between cancer-death and accidental-death groups. Mean and standard error for nerve fibre layer (NFL) network, retinal ganglion cell (RGC) layer network, inner plexiform layer and superficial inner nuclear layer

(IPL/superficial INL) network and deep inner nuclear layer and outer plexiform layer (deep INL/OPL) network is provided.

Capillary density in the Deep INL/OPL network was significantly different between the two groups. 


\section{$\underline{\text { References }}$}

1. Attwell D, Buchan AM, Charpak S et al. Glial and neuronal control of brain blood flow. Nature. 2010;468:232-243.

2. Gordon GR, Choi HB, Rungta RL, Ellis-Davies GC, Macvicar BA. Brain metabolism dictates the polarity of astrocyte control over arterioles. Nature. 2008;456:745-749.

3. Yu D-Y, Cringle SJ, Alder VA, Su EN. Intraretinal oxygen distribution in rats as a function of systemic blood pressure. Am J Physiol. 1994;36:H2498-H2507.

4. Yu D-Y, Cringle SJ. Oxygen distribution in the mouse retina. Invest Ophthalmol Vis Sci. 2006;47:1109-1112.

5. Yu DY, Cringle SJ, Su EN. Intraretinal oxygen distribution in the monkey retina and the response to systemic hyperoxia. Invest Ophthalmol Vis Sci. 2005;46:4728-4733.

6. Cringle SJ, Yu PK, Su EN, Yu DY. Oxygen distribution and consumption in the developing rat retina. Invest Ophthalmol Vis Sci. 2006;47:4072-4076.

7. Yu D-Y, Cringle SJ, Su E-N, Yu PK. Retinal degeneration and local oxygen metabolism. Exp Eye Res. 2005;80:745-751.

8. Yu DY, Cringle SJ, Yu PK, Su EN. Intraretinal oxygen distribution and consumption during retinal artery occlusion and graded hyperoxic ventilation in the rat. Invest Ophthalmol Vis Sci. 2007;48:2290-2296.

9. Yu D-Y, Cringle SJ. Oxygen distribution and consumption within the retina in vascularised and avascular retinas and in animal models of retinal disease. Prog Retina Eye Res. 2001;20:175-208.

10. Gariano RF, Iruela-Arispe ML, Hendrickson AE. Vascular development in primate retina: comparison of laminar plexus formation in monkey and human. Invest Ophthalmol Vis Sci. 1994;35:3442-3455.

11. Yu PK, Balaratnasingam C, Cringle SJ et al. Microstructure and network organization of the microvasculature in the human macula. Invest Ophthalmol Vis Sci. 2010;51:6735-6743.

12. Yu PK, Balaratnasingam C, Morgan WH et al. The structural relationship between the microvasculature, neurons, and glia in the human retina. Invest Ophthalmol Vis Sci. 2010;51:447-458.

13. Yu PK, Tan PE, Morgan WH et al. Age-related changes in venous endothelial phenotype at human retinal artery-vein crossing points. Invest Ophthalmol Vis Sci. 2012;53:1108-1116.

14. Hogan MJ, Alvarado JA, Esperson Weddell J. Histology of the Human Eye: An Atlas and Textbook. Philadelphia, W.B. Saunders Company;1971

15. Snodderly DM, Weinhaus RS. Retinal vasculature of the fovea of the squirrel monkey, Saimiri sciureus: Three-dimensional architecture, visual screening, and relationships to the neuronal layers. Journal of Comparative Neurology. 1990;297:145-163.

16. Snodderly DM, Weinhaus RS, Choi JC. Neural-vascular relationships in central retina of macaque monkeys (Macaca fascicularis). J Neurosci. 1992;12:1169-1193.

17. Gariano RF, Iruela-Arispe ML, Sage EH, Hendrickson AE. Immunohistochemical characterization of developing and mature primate retinal blood vessels. Invest Ophthalmol Vis Sci. 1996;37:93-103.

18. Xiao M, Hendrickson A. Spatial and temporal expression of short, long/medium, or both opsins in human fetal cones. Journal of Comparative Neurology. 2000;425:545-559. 
19. Surgucheva I, Weisman AD, Goldberg JL, Shnyra A, Surguchov A. Gamma-synuclein as a marker of retinal ganglion cells. Mol Vis. 2008;14:1540-1548.

20. Haverkamp S, Haeseleer F, Hendrickson A. A comparison of immunocytochemical markers to identify bipolar cell types in human and monkey retina. Visual Neuroscience. 2003;20:589-600.

21. Rackl A, Pawlik G, Bing RJ. Cerebral capillary topography and red cell flow in vivo. In: Ceros-Navaroo J, Fritschka E, eds. Cerebral Microcirculation and Metabolism. New York: Raven Press; 1981:17-21.

22. Richard E, van Gool WA, Hoozemans JJ et al. Morphometric changes in the cortical microvascular network in Alzheimer's disease. J Alzheimers Dis. 2010;22:811-818.

23. Shaver SW, Pang JJ, Wall KM, Sposito NM, Gross PM. Subregional topography of capillaries in the dorsal vagal complex of rats: I. Morphometric properties. J Comp Neurol. 1991;306:73-82.

24. Cerebral Circulation. A critical discussion of the symposium. In: Cobb S, eds. The Circulation of the Brain and Spinal Cord. Baltimore: Williams and Wilkins; 1938:710-752.

25. R Development Core Team. R: A Language and Environment for Statistical Computing. 2011. Vienna, Austria, R Foundation for Statistical Computing.

26. Yu DY, Cringle SJ, Alder VA, Su EN, Yu PK. Intraretinal oxygen distribution and choroidal regulation in the avascular retina of guinea pigs. Am J Physiol. 1996;270:H965-H973.

27. Yu D-Y, Cringle SJ. Low oxygen consumption in the inner retina of the visual streak of the rabbit. Am J Physiol (Heart C). 2004;286:H419-H423.

28. Yu D-Y, Cringle SJ, Su E-N et al. Laser induced changes in intraretinal oxygen distribution in pigmented rabbits. Invest Ophthalmol Vis Sci. 2005;46:988-999.

29. Yu D-Y, Cringle SJ, Su EN, Yu PK. Retinal oxygen distribution in a rat model of retinal ischemia. Invest Ophthalmol Vis Sci. 2000;41:S19

30. Danis RP, Wallow IHL. HRP/Trypsin technique for studies of the retinal vasculature. Invest Ophthalmol Vis Sci. 1986;27:434-437.

31. Bek T, Jensen PK. Three-dimensional structure of human retinal vessels studied by vascular casting. Acta Ophthalmol (Copenh). 1993;71:506-513.

32. Mendis KR, Balaratnasingam $\mathrm{C}, \mathrm{Yu} \mathrm{P}$ et al. Correlation of histologic and clinical images to determine the diagnostic value of fluorescein angiography for studying retinal capillary detail. Invest Ophthalmol Vis Sci. 2010;51:5864-5869.

33. Cheng H, Nair G, Walker TA et al. Structural and functional MRI reveals multiple retinal layers. Proc Natl Acad Sci U S A. 2006;103:17525-17530.

34. Toussaint D, Kuwabara T, Cogan DG. Retinal vascular patterns. II Human retinal vessels studied in three dimensions. Arch Ophthalmol. 1961;65:137-143.

35. Michaelson IC, Campbell ACP. The anatomy of the finer retinal vessels, and some observations on their significance in certain retinal diseases. Trans Ophthalmol Soc UK. 1940;60:71-111.

36. Craigie EH. The vascularity of the cerebral cortex of the albino rat. J Comp Neurol. 1921;33:193-212.

37. Motti ED, Imhof HG, Yasargil MG. The terminal vascular bed in the superficial cortex of the rat. An SEM study of corrosion casts. J Neurosurg. 1986;65:834-846.

38. Bell MA, Ball MJ. Morphometric comparison of hippocampal microvasculature in ageing and demented people: diameters and densities. Acta Neuropathol. 1981;53:299-318. 
39. Pawlik G, Rackl A, Bing RJ. Quantitative capillary topography and blood flow in the cerebral cortex of cats: an in vivo microscopic study. Brain Res. 1981;208:35-58.

40. Tuor UI, Kurpita G, Simone C. Correlation of local changes in cerebral blood flow, capillary density, and cytochrome oxidase during development. J Comp Neurol. 1994;342:439-448.

41. Weber B, Keller AL, Reichold J, Logothetis NK. The microvascular system of the striate and extrastriate visual cortex of the macaque. Cereb Cortex. 2008;18:2318-2330.

42. Kergoat H, Herard ME, Lemay M. RGC sensitivity to mild systemic hypoxia. Invest Ophthalmol Vis Sci. 2006;47:5423-5427. 


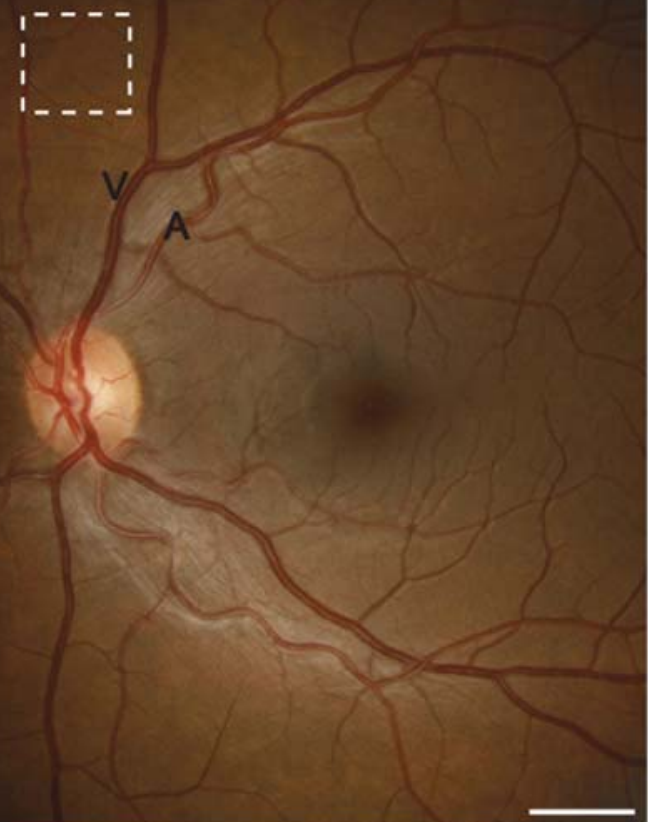

B

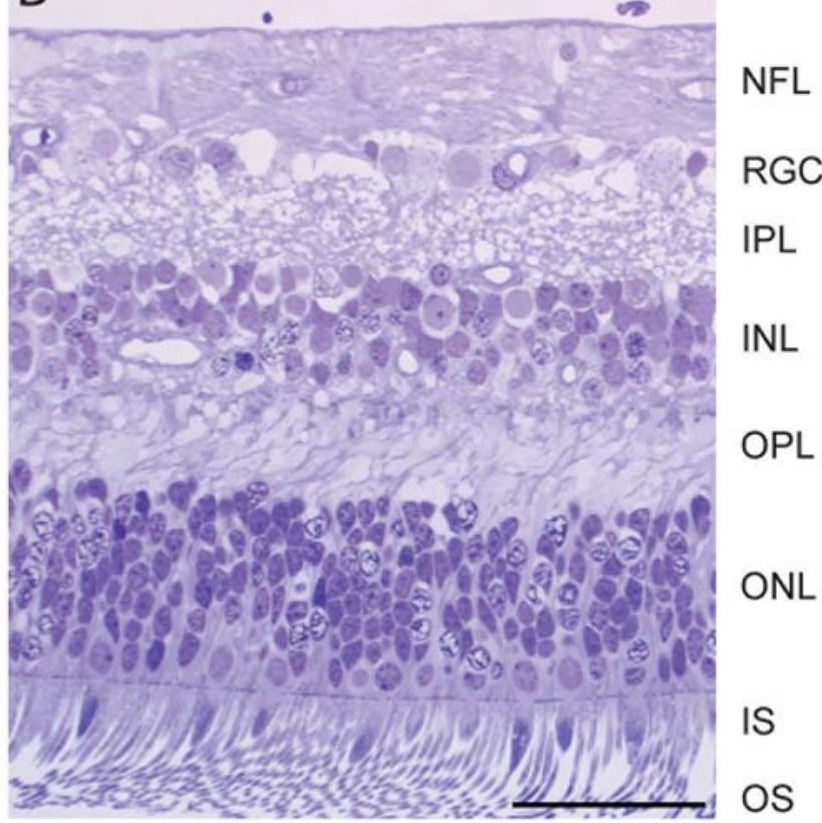



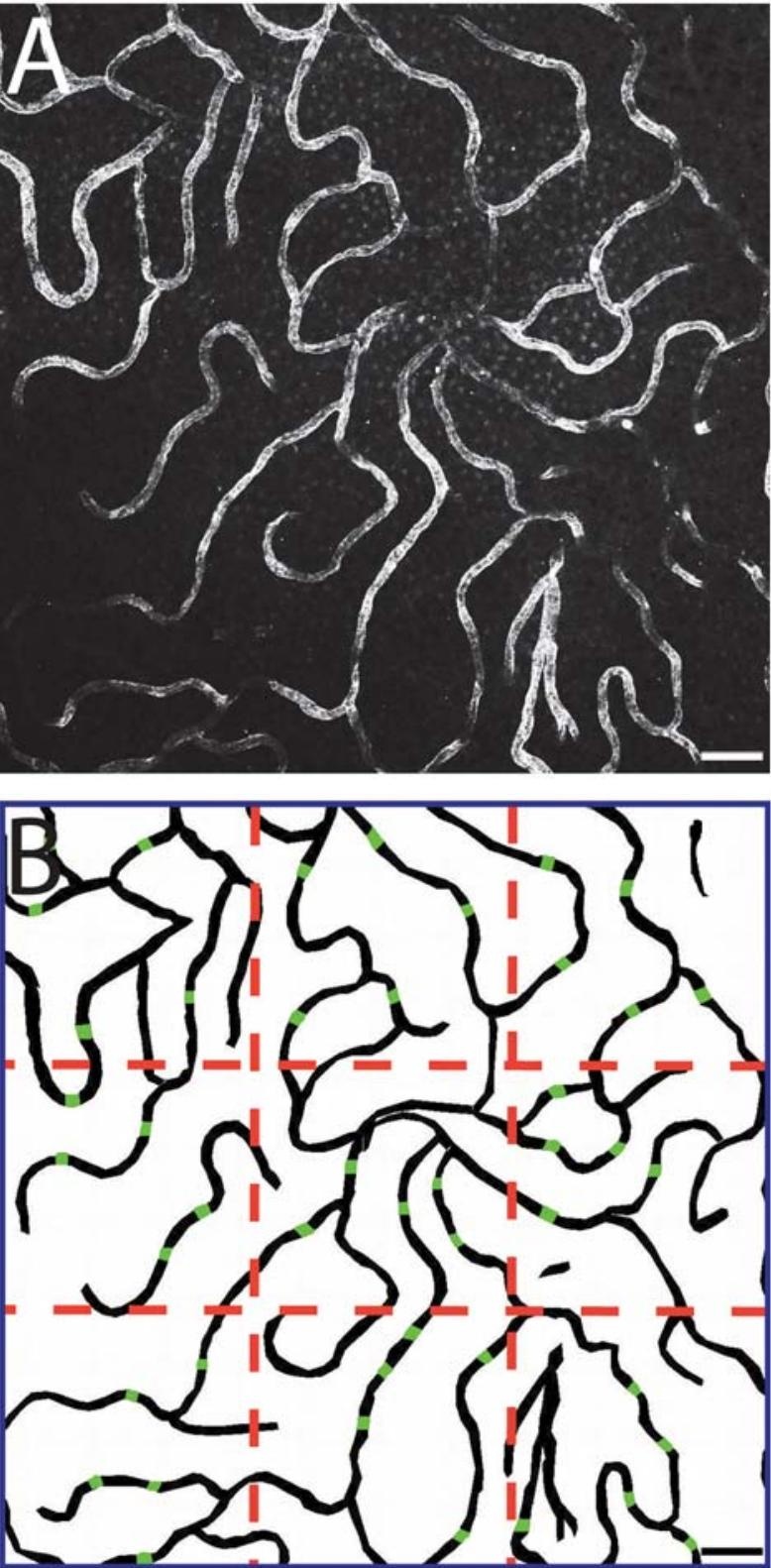


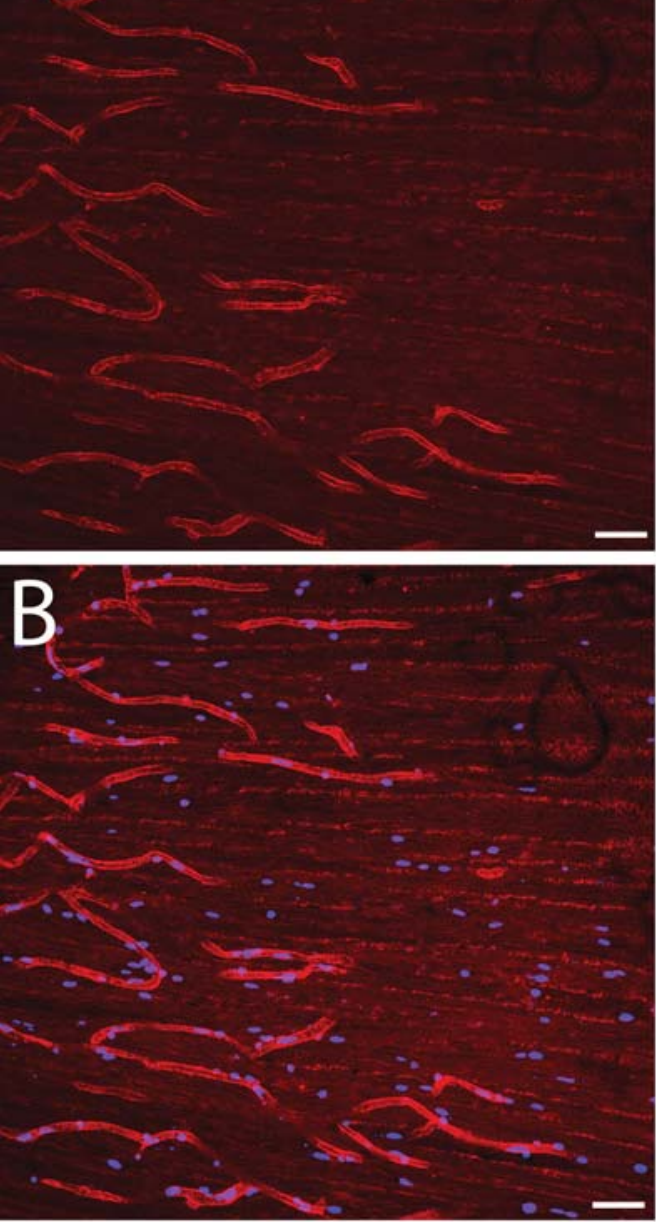

\section{VITREOUS}

\section{Lectins}

Hoechst

y-synuclein

NFL RGC IPL INL OPL ONL 


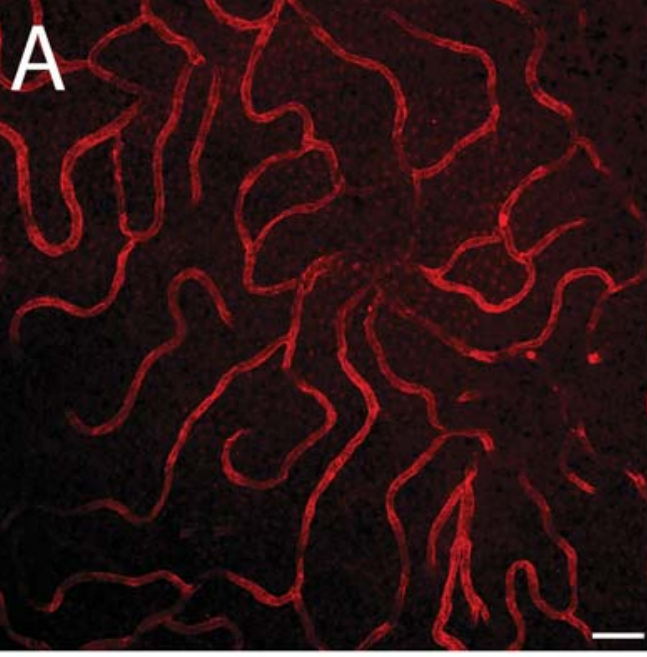

\section{B}

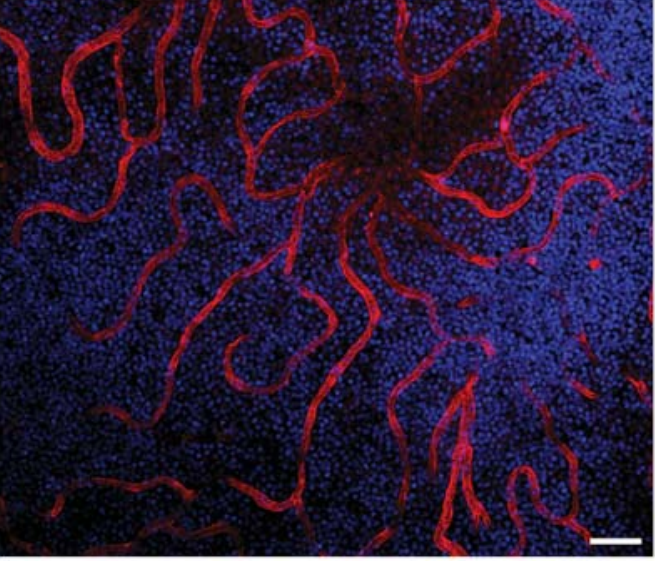

C

VITREOUS

Lectins

Hoechst

Parvalbumin

NFL

RGC IPL INL

OPL

$\mathrm{ONL}$

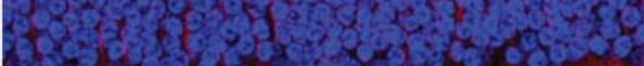

